\title{
Hennemann, Moritz, Interaktion und Partizipation. Dimensionen systemischer Bindung im Privatrecht.
}

\author{
Mohr Siebeck Verlag, Tübingen, 2020. XXIV, 430 Seiten. ISBN \\ 978-3-16-159574-5
}

\section{Christian Armbrüster}

Angenommen: 4. Februar 2021 / Online publiziert: 11. Februar 2021

(C) Der/die Autor(en) 2021

Publikationen zur digitalen Transformation der Privatrechtsgesellschaft haben derzeit Konjunktur. Dabei steht häufig die Frage im Mittelpunkt, inwieweit das Vertragsrecht herkömmlicher Prägung den Herausforderungen der Digitalisierung noch gewachsen ist. Hennemann nimmt in seiner Untersuchung, einer Freiburger Habilitationsschrift, drei Entwicklungen genauer in den Blick: Digitale Plattformen als Intermediäre für den Vertragsschluss, die technisch basierte Selbstorganisation Privater unter Wegfall von Mittelsleuten wie Banken oder Treuhändern (etwa durch Blockchain-Technologie) und die Einbindung automatisierter und autonomer Anwendungen in den Vertragsschlussprozess, wodurch womöglich neue KI-basierte Handlungssubjekte entstehen.

Es ist unschwer zu erkennen, dass alle drei Entwicklungen auch im Versicherungssektor bereits verschiedentlich präsent sind. Um nur einige Beispiele zu nennen: Eine immer größere Zahl von Versicherungsverträgen wird über Internet-Plattformen geschlossen; Blockchain-Technologie spielt insbesondere in der Transport- und in der Rückversicherung eine zunehmend wichtige Rolle; bei der Entscheidung über den Vertragsschluss werden vermehrt Algorithmen eingesetzt. Den Autor interessieren insbesondere die Änderungen, die sich aus all diesen Entwicklungen - namentlich aus der Einbindung Dritter in die klassische bipolare Vertragsbeziehung, der Mehrzahl von Verträgen mit verschiedenen Partnern und dem Auftreten neuer, nämlich digitaler Akteure beim Vertragsschluss - für das Vertragsrecht ergeben.

Die Arbeit bietet zunächst einen guten Überblick zu den tatsächlichen Entwicklungen (S. 21 ff.). Dabei führt Hennemann den Begriff „,systemische Bindung“ ein, womit die - graduell unterschiedliche - Einbindung Dritter in das Ob und Wie der vertraglichen Bindung gemeint ist. Die verschiedenen Erscheinungsformen dieser

C. Armbrüster $(\square)$

Fachbereich Rechtswissenschaft, Freie Universität Berlin, Van't-Hoff-Str. 8, 14195 Berlin,

Deutschland

E-Mail: c.armbruester@fu-berlin.de 
Einbindung werden sodann näher beleuchtet (S. $45 \mathrm{ff}$.). Es folgen in drei größeren Kapiteln ein rechtstheoretischer, ein rechtsdogmatischer und ein rechtspraktischer Diskurs, bevor abschließend Fortentwicklungspotentiale erörtert werden. Zur Rechtstheorie stellt Hennemann zunächst die wesentlichen Ansätze vor, um daraus zusammenfassend das Petitum herzuleiten, es müsse ,der strukturellen Gefahr entgegengewirkt werden, dass die Partizipation Dritter in einer rein zweipoligen Betrachtung marginalisiert“ (S. 96) wird. Maßstab für die anschließende vertragsrechtsdogmatische Analyse soll es demnach sein, den Erwartungen aller Akteure gerecht zu werden.

Der Rechtsdogmatik widmet der Autor sodann den größten Teil seiner Untersuchung (S. $99 \mathrm{ff}$.). Dabei knüpft er zunächst unter dem Schlagwort „Computererklärungen“" an die lebhafte Diskussion darüber an, wie automatisch generierte Erklärungen in den Kategorien der Rechtsgeschäftslehre des BGB einzuordnen sind. Insoweit folgt er weitgehend der Linie, dass die allgemeinen Regeln zum Vertragsschluss anwendbar sind und dass durch den Einsatz von Plattformen der Grundsatz der Relativität der Schuldverhältnisse nicht durchbrochen wird. Interessant ist der Vergleich von Bausteinverträgen mit mehreren Vertragspartnern mit dem Pauschalreisevertrag; in beiden Fällen seien die Erwartungen des Kunden auf ein „Gesamtpaket“ gerichtet, so dass die Grenze zwischen reiner Vermittlung und eigener Verpflichtung des Vermittlers fließend werde (S. $151 \mathrm{f}$.).

Bei der Blockchain als einem dezentralen Kooperationsmechanismus sieht Hennemann die rechtliche Grundlage regelmäßig in einer Innengesellschaft bürgerlichen Rechts (S. 183 ff.). Um die Vertragsstrukturen auf der Blockchain selbst zu erfassen, brauche es keine eigenständige Figur eines Vertragsnetzwerks. Den Erwartungen der Nutzer lasse sich durch das Instrument der (ergänzenden) Vertragsauslegung Rechnung tragen. Die von autonomen Agenten, also mittels Künstlicher Intelligenz, abgegebenen Erklärungen lassen sich nach Ansicht des Autors nicht mit den Regeln der Computererklärung adäquat erfassen. Er verwirft die verschiedenen $\mathrm{Zu}-$ rechnungsmodelle und hält es de lege ferenda für erwägenswert autonome Agenten als Vertragsschlussgehilfen einzuordnen (S. $338 \mathrm{ff}$.). Zudem plädiert er für die regulatorische Begründung eines Rechtsstatus für autonome Anwendungen und für die Schaffung eines Vertragssicherheitsrechts, unter anderem indem für autonome Agenten eine Zertifizierung eingeführt wird (S. $379 \mathrm{ff}$.).

Das Werk spricht eine enorme Bandbreite von Einzelthemen an; dabei bleibt manches zwangsläufig im Ungefähren. Dies gilt auch für einige der rechtsdogmatischen Erwägungen und insbesondere für die Perspektiven künftiger Entwicklungen. Für den versicherungswissenschaftlich interessierten Leser bietet das Buch gleichwohl eine Fülle interessanter Informationen und Denkanstöße. Beispielhaft seien etwa die Ausführungen zu den Aktivitäten der Europäischen Kommission und des Europäischen Parlaments im Bereich der Künstlichen Intelligenz genannt (S. $309 \mathrm{ff}$.) - eine Entwicklung, die unter dem Schlagwort „Digital Finance Strategy“ bereits in naher Zukunft Regulierungen erwarten lässt, die für den Versicherungssektor von großer Bedeutung sein werden.

Funding Open Access funding enabled and organized by Projekt DEAL. 
Open Access Dieser Artikel wird unter der Creative Commons Namensnennung 4.0 International Lizenz veröffentlicht, welche die Nutzung, Vervielfältigung, Bearbeitung, Verbreitung und Wiedergabe in jeglichem Medium und Format erlaubt, sofern Sie den/die ursprünglichen Autor(en) und die Quelle ordnungsgemäß nennen, einen Link zur Creative Commons Lizenz beifügen und angeben, ob Änderungen vorgenommen wurden.

Die in diesem Artikel enthaltenen Bilder und sonstiges Drittmaterial unterliegen ebenfalls der genannten Creative Commons Lizenz, sofern sich aus der Abbildungslegende nichts anderes ergibt. Sofern das betreffende Material nicht unter der genannten Creative Commons Lizenz steht und die betreffende Handlung nicht nach gesetzlichen Vorschriften erlaubt ist, ist für die oben aufgeführten Weiterverwendungen des Materials die Einwilligung des jeweiligen Rechteinhabers einzuholen.

Weitere Details zur Lizenz entnehmen Sie bitte der Lizenzinformation auf http://creativecommons.org/ licenses/by/4.0/deed.de. 